Discussion Paper No. 991

\title{
MODELLING REALIZED VOLATILITY \\ IN ELECTRICITY SPOT PRICES: \\ NEW INSIGHTS AND APPLICATION \\ TO THE JAPANESE ELECTRICITY MARKET
}

\author{
Aitor Ciarreta \\ Peru Muniain \\ Ainhoa Zarraga
}

February 2017

The Institute of Social and Economic Research

Osaka University

6-1 Mihogaoka, Ibaraki, Osaka 567-0047, Japan 


\title{
Modelling Realized Volatility in Electricity Spot Prices: New insights and Application to the Japanese Electricity Market
}

\author{
Aitor Ciarreta, Peru Muniain†, Ainhoa Zarraga ${ }^{\ddagger}$ \\ University of the Basque Country, Spain
}

February 24, 2017

\begin{abstract}
The paper analyzes volatility of the electricity prices in the Japanese day-ahead market using realized volatility. We use several jump tests to decompose total realized variation into jump and continuous components. Then, we estimate several HAR models that show the time-dependence structure of the volatility. Our results show that even though that market is narrow, it is relevant to identify jumps in volatility. Besides, modeling residuals improve estimation results. The timedependent structure of the prices is present in volatility as well.
\end{abstract}

\section{Introduction}

Two decades ago, the electricity sector worldwide used to be organized as a vertically integrated industry in which prices were set on a cost-of-service regulation. In this setting, prices used to change on a yearly basis. ${ }^{1}$ Deregulation has implied the launching of pool markets in which selling bids and purchase orders meet to determine the equilibrium price and quantity for each time interval of the following day. There is a considerable and increasing amount of trading in both the spot and derivative markets that have emerged depending on the maturity of the market.

The nature of electricity makes the market price more sensitive to demand and supply changes. The resulting high level of price volatility and ocasional price jumps experienced in these markets has allowed the development of instruments to hedge market agents against risk involved. In this respect, empirical evidence shows that volume traded is

\footnotetext{
${ }^{*}$ Email: aitor.ciarreta@ehu.eus. I would like to thank financial support from Ministerio de Economía y Competitividad under research ECO2015-64467-R and from Dpto. de Educación, Universidades e Investigación del Gobierno Vasco under research grant IT-783-13. I would like to thank the Institute of Social and Economic Research, University of Osaka, for financial aid.

${ }^{\dagger}$ Email: peru.muniain@ehu.eus.

${ }^{\ddagger}$ Email: ainhoa.zarraga@ehu.eus.

${ }^{1}$ Most of the countries updated prices according to the inflation rate observed the year before.
} 
negative correlated with price variation. ${ }^{2}$ Power suppliers and retailers seek to manage their portfolios in this volatile environment. Understanding price volatility is important for agents to design their bidding strategies optimally. Development of intraday markets and financial contracts for future delivery of power is the result of agents seeking to effectively manage risk associated to short-term day-ahead transactions. Thus, electricity markets, as other financial markets, are evolving to allow participants to develope effective risk management strategies. The more electricity markets evolve the larger the amount of risk that has to be managed and controlled by market agents.

Models that incorporate the stochastic nature of volatility have developed rapidly following initial works by Hull and White (1987) and Hull (2000). Applications to electricity prices can be found in Deng (1999), Escribano et al (2011), Lucia and Schwartz (2002), Skantze et al. (2000), Geman and Roncoroni (2006) among others.

Our interest is to use the information contained in within-day prices to analyze and forecast volatility. Variation of asset prices is measured by the sum of squared returns over defined time periods. The mathematics behind is based on the quadratic variation process (Back, 1991). Increments of the risk premium are associated to increments of quadratic variation in asset pricing theory. The estimation of quadratic variation using realized volatility measures was initially discussed in the works of Andersen and Bollerslev (1998), Comte and Renault (1998), and Bandorff-Nielsen and Shephard (2002). Since then, the estimation of daily volatility using realized measures relying on high frequency intraday data has stimulated a growing field of volatility modeling and estimation.

The development of nonparametric methods for volatility estimation by BandorffNielsen and Shephard (2004) offer the possibility to estimate separately the diffusive and the jump components of the price process using realised and bipower variation. Huang and Tauchen (2005) and Andersen et al. (2007) showed that jumps play an important role in the movement of prices. Since then, non-parametric jump tests using realised measures have been proposed attending the particular features of the stochastic process. All of them consider jumps as rare events and try to extract the jumps from the continuous part of the volatility. The literature has grown rapidly since the works by Barndorff-Nielsen and Shephard (BNS, 2006). Further developments by Andersen et al. (ABD, 2007), Lee and Mykland (LM, 2008), Ait-Sahalia and Jacod (2008), Jiang and Oomen (JO, 2008), Corsi et al (CPR, 2010), Podolskij and Ziggel (2010) and Andersen et al. (ABD, 2012), among others have been also proposed, simulated and tested.

There are not that many applications of realized volatility to electricity markets. The are several characteristics of the data generating process to consider when modelling realized volatility in electricity prices. First, the non-storability nature of electricity (except for hydroelectric generation) which forces prices to be highly dependent on spot demand and supply conditions. Second, strong seasonal effects, mostly observed yearly, weekly and daily periodic cycles. Third, presence of frequent price spikes coupled with

\footnotetext{
${ }^{2}$ Examples of the negative and significant correlation between price volatility (measured by the daily standard deviation) and quantity traded are -0.0494 in the Japanese market, -0.1889 in EPEXGermany, -0.3662 in EPEX-Switzerland, and -0.1709 in the Spanish-Portuguese market.
} 
non-Gaussian characteristics of the implied probability distribution of the day-ahead prices. Therefore, proper statistical modelling of spot prices is necessary for optimal valuation of financial products and optimal physical assets management in the electricity sector.

Although each estimator has its own properties, there is no single method which is preferred to the rest. Two select the best model we use two approaches: (1) In-sample goodness of fit and (2) a test for bias in the estimation.

Several studies using Heterogenous Autorregressive (HAR) estimation to different specifications of realized volatility models in electricity markets are present in the literature. Ciarreta and Zarraga (2016) for the sequential intraday market, Qu at el (2016) propose a logistic beta smooth transition heterogeneous autoregressive (LSTHAR) model of realized volatility outperforms other HAR models based on Corsi (2009). To our knowledge, there are not studies on the Japanese market using statistical models of price formation and volatility.

Several contributions are in the paper. First, we propose a departure from the existing literature on how seasonality is removed in the returns. We smooth the time series of prices using a robust non-linear smoother by Velleman (1980). The smooth technique is easy to implement. Second, once seasonality is removed, we decompose realized volatility following BNS (2006), ABD (2007), CPR (2010) and ABD-LM (2012). At this point we propose a modified version of the ABD-LM test in which the local volatility is estimated using a kernel approach. We propose a discrete Weibull distribution. Then, we estimate the bias of the different decomposition statistics following Fang et al. (2012). And third, a minor contribution which is the study of volatility in the Japanese electricity market that has not been addressed before, basically because the market only trades $2 \%$ of the total consumption.

The paper is organized as follows. In Section 2, we review the time series process model and the nonparametric tests based on realized measures. Section 3 briefly describes the Japanese electricity market structural, characteristics and regulation. Section 4 describes the data. Section 5 reports empirical results and discusses the model selection criteria. Section 5 proposes new lines of research. Section 6 concludes.

\section{Methodology}

\subsection{Jump tests}

Assume the dynamics of the (logarithmic) prices follow a jump-diffusion process which contains a non-stochastic component and a stochastic component of the form

$$
d p_{t}=\mu_{t} d t+\sigma_{t} d W_{t}+d J_{t}
$$

where $\mu_{t}$ is the drift, $\sigma_{t}$ is the diffusion parameter, $W_{t}$ is a standard Brownian motion and $J_{t}$ is the jump process such that $J_{t}=\sum_{j=1}^{N_{t}} c_{t_{j}}$, where $c_{t_{j}}$ is the size of the jump and $N_{t}$ is the number of jumps up to time $t$. Jumps are assumed to be countable rare events that migh happen along the path of the continuous price process. The quadratic 
variation of the process $\left(Q V_{t}\right)$ has a continuous part corresponding to the integrated volatility or integrated variance $\left(I V_{t}\right)$, which is the continuous component, and the sum of the squares of the jump sizes, which is the discontinuous or jump component. Thus $Q V_{t}$ is the sum of both components,

$$
Q V_{t}=\int_{0}^{t} \sigma_{s}^{2} d s+\sum_{j=1}^{N_{t}} c_{t_{j}}^{2} .
$$

Assume we divide the time interval $[0, t]$ into $M$ subintervals with length $\Delta=1 / M$ where $M$ is taken constant for each $t$ (as $\Delta \rightarrow 0$ frequency of interavals increases). Overall, there are $T$ trading periods observed with the same time interval. Therefore, following Andersen and Bollerslev (1998), realized volatility for day $t, R V_{t}$, is defined as the sum of the squares of intraday returns,

$$
R V_{t}=\sum_{j=1}^{M} r_{t, j}^{2},
$$

where $r_{t, j}$ is the intraday log-price difference at $j, r_{t, j}=p_{t, j}-p_{t, j-1}$, which is the usual definition of return in financial markets. ${ }^{3}$ Under the null of absence of jumps, $Q V_{t}$ is consistently estimated by $R V_{t}$. Therefore, $R V_{t}$ is taken as a measure for the unobserved volatility of a high-frequency time series.

$I V_{t}$ captures the continuous, predictable component of $R V_{t}$ and it can be estimated using different methods. In this paper, we use several jump test statistics that have been proved to converge to a normal distribution when $M \rightarrow \infty$. Hence, if the statistic exceeds the critical value $\Phi_{1-\alpha}$, day $t$ is classified as a jump day. For a chosen significance level $\alpha$, the jump component of volatility at day $t$ is obtained as:

$$
J V_{t}=I_{Z_{t}>\Phi_{1-\alpha}}\left(R V_{t}-\widehat{I V}_{t}\right),
$$

where $Z_{t}$ is the jump test statistic and $\widehat{I V}_{t}$ the corresponding estimator of $I V_{t}$ obtained with each method, and $I_{Z_{t}>\Phi_{1-\alpha}}$ is 1 if $Z_{t}>\Phi_{1-\alpha}$, and 0 otherwise. The $Z_{t}$ test statitic is the one proposed by Huang and Tauchen (2005) which is a class of Hausman (1978) type of test. To ensure $J V_{t}>0$ it is defined as $J V_{t}=\max \left(R V_{t}-\widehat{I V}_{t}, 0\right)$. Once jump variation $\left(J V_{t}\right)$ is estimated, the continuous component of the total variation, $C V_{t}$, is given by the difference between the two, $C V_{t}=R V_{t}-J V_{t}$.

In this paper, existing non-parametric jump-robust type estimators are grouped into two categories. The first category includes those jump tests that enable us to estimate $J V_{t}$ and $C V_{t}$ from total $R V_{t}$, these are BNS (2006), Andersen, Dobrev and Schaumburg (2009), and CPR (2010). The second category includes those tests that do not allow us

\footnotetext{
${ }^{3}$ As we show in the descriptive statistics, in the Japanese market, the minimum $p_{t, j}$ is strictly positive. Therefore, we can define realized volatility in terms of returns. However, in many electricity markets $p_{t, j}$ can be zero or negative thus either up-scaling logarithmic transformations of prices are considered or the price process is taken as such, and realized volatility is defined in terms of price differences.
} 
to directly estimate $J V_{t}$ and $C V_{t}$ from total $R V_{t}$ but only identify jump-times, these are ABD, (2007), and LM, (2008) and JO, (2008).

\section{Tests based on multipower variation:}

BNS (2006) test is based on the use of the realised bipower variation. They proof (Theorem 1) how $I V_{t}$ can be consistently estimated using bipower variation $\left(B V_{t}\right)$. The 1, 1-order $B V_{t}$ process, if it exists, is defined as $^{4}$

$$
B V_{t}=\mu_{1}^{2} \frac{M}{(M-1)} \sum_{j=2}^{M}\left|r_{j}\right|\left|r_{j-1}\right|,
$$

where $\mu_{1}=E|u|=\sqrt{2} / \sqrt{\pi}$. Following Huang and Tauchen (2005), the test statistic, called $Z_{t}^{B N S}$, is based on the difference between $R V_{t}$ and the jump-robust measure of variance, $B V_{t}$, to detect significant jumps:

$$
Z_{t}^{B N S}=\sqrt{M} \frac{\left(R V_{t}-B V_{t}\right) / R V_{t}}{\sqrt{\left(\frac{\pi^{2}}{4}+\pi-5\right) \max \left[1, T Q_{t} / B V_{t}^{2}\right]}}
$$

The denominator represents the effect of integrated quarticity, which can be estimated using the tripower quarticity,

$$
T Q_{t}=1.74\left(\frac{M^{2}}{M-2}\right) \sum_{j=3}^{M}\left(\left|r_{j}\right|\left|r_{j-1}\right|\left|r_{j-2}\right|\right)^{4 / 3}
$$

As the sampling frequency increases, $\Delta \rightarrow 0$, the impact of jumps is reduced because jump-returns are multiplied by adjacent non-jump returns. In finite samples, it can be shown that $B V_{t}$ is upward biased in the presence of jumps and as a result the jump component is underestimated. Besides, $B V_{t}$ is also affected by zero returns, thus reducing its value and consequently detecting more jumps when returns are zero or close to zero.

ABD, (2012) propose two jump-robust consistent estimators of $I V$ using the nearest neighbour truncation. These are the $M i n R V_{t}$ and $M e d R V_{t}$ estimators.

$$
\begin{aligned}
& \operatorname{MinRV}_{t}=2.75\left(\frac{M}{M-2}\right) \sum_{j=2}^{M} \min \left(\left|r_{t, j}\right|,\left|r_{t, j-1}\right|\right)^{2} \\
& M e d R V_{t}=1.42\left(\frac{M}{M-2}\right) \sum_{j=2}^{M-1} \operatorname{med}\left(\left|r_{t, j-1}\right|,\left|r_{t, j}\right|,\left|r_{t, j+1}\right|\right)^{2}
\end{aligned}
$$

The $\operatorname{MinR} V_{t}$ is exposed to zero returns and is less efficient than the $M e d R V_{t}$ estimator which is based on the median on blocks of three consecutive returns. These two estimators can be used to construct the jump test statistic following Huang and Tauchen (2005):

\footnotetext{
${ }^{4}$ BNS (2004) extend the properties of $B V_{t}$ for the products of $k$-lag length absolute returns for $k \geq 1$. Because we want to make our results comparable for the different tests, we restrict to the case of $k=1$. Taking $k>1$ allows for correction for serial correlation. For instance, Ullrich (2012) analyzes the case from $k=1$ to $k=8$.
} 


$$
\begin{aligned}
Z_{t}^{M i n R V} & =\sqrt{M} \frac{\left(R V_{t}-M i n R V_{t}\right) / R V_{t}}{\sqrt{1.81 \max \left[1, \frac{M i n R Q_{t}}{M i n R V_{t}^{2}}\right]}} \stackrel{L}{\rightarrow} N(0,1) \\
Z_{t}^{M e d R V} & =\sqrt{M} \frac{\left(R V_{t}-M e d R V_{t}\right) / R V_{t}}{\sqrt{0.96 \max \left[1, \frac{M e d R Q_{t}}{M e d R V_{t}^{2}}\right]}} \stackrel{L}{\rightarrow} N(0,1)
\end{aligned}
$$

where the estimator of the integrated quarticities in the denominators are

$$
\begin{aligned}
\operatorname{MinR} Q_{t} & =2.21 \frac{M^{2}}{M-1} \sum_{j=2}^{M} \min \left(\left|r_{t, j}\right|,\left|r_{t, j-1}\right|\right)^{4} \\
M e d R Q_{t} & =0.92 \frac{M^{2}}{M-2} \sum_{j=2}^{M-1} \operatorname{med}\left(\left|r_{t, j-1}\right|,\left|r_{t, j}\right|,\left|r_{t, j+1}\right|\right)^{4},
\end{aligned}
$$

respectively. ABD (2012) argue that increasing the block length to any $k>1$ in the estimation of $I V_{t}$ reduces efficiency. Therefore, we restrict all the jump tests for the $I V$ estimators based on adjacent returns. ${ }^{5}$

CPR, (2010) propose a consistent and nearly unbiased estimator of $I V_{t}$ based on threshold bipower variation:

$$
C T B P V_{t}=1.57 \sum_{j=2}^{M} Z_{1}\left(r_{j}, \vartheta_{j}\right) Z_{1}\left(r_{j-1}, \vartheta_{j-1}\right),
$$

where the function $Z_{1}$ is defined as:

$$
Z_{1}\left(r_{j}, \vartheta_{j}\right)= \begin{cases}\left|r_{j}\right| & \text { if } r_{j}^{2} \leq \vartheta_{j} \\ 1.094 \vartheta_{j}^{1 / 2} & \text { if } r_{j}^{2}>\vartheta_{j}\end{cases}
$$

which depends on the returns and the value of the threshold $\vartheta_{j}=c_{\vartheta}^{2} \hat{V}_{j}^{Z}$. The local volatility estimate, $\hat{V}_{j}^{Z}$, is estimated following an iterative process using a Gausssian kernel as

$$
\widehat{V}_{j}^{Z}=\frac{\sum_{i=-L, i \neq-1,0,1}^{L} K\left(\frac{i}{L}\right) r_{j+i}^{2} I_{\left\{r_{j+i}^{2} \leq c_{v}^{2} \widehat{V}_{j}^{Z-1}\right\}}}{\sum_{i=-L, i \neq-1,0,1}^{L} K\left(\frac{i}{L}\right) I_{\left\{r_{j+i}^{2} \leq c_{v}^{2} \widehat{V}_{j}^{Z-1}\right\}}} \text { for } Z=1,2, \ldots
$$

\footnotetext{
${ }^{5}$ In the appendix we also report results for both estimators taking one block jump although results should be taken cautiously due to data frequency.
} 
As they suggest, we consider $c_{\vartheta}=3$ and the number of iterations needed to converge depends on the process itself. ${ }^{6}$ The choice of the threshold does not affect the robustness of the $I V_{t}$ estimator, and its impact on estimation is marginal. The corresponding jump test statistic is also based on Huang and Tauchen (2005):

$$
Z_{t}^{C T B P V}=\sqrt{M} \frac{\left(R V_{t}-C T B P V_{t}\right) / R V_{t}}{\sqrt{\left(\frac{\pi^{2}}{4}+\pi-5\right) \max \left[1, \frac{C T T r i P V_{t}}{C T B P V_{t}^{2}}\right]}}
$$

where $C T$ TriPV $V_{t}=1.74 M \sum_{j=3}^{M} \prod_{k=1}^{3} Z_{4 / 3}\left(r_{j-k+1}, \vartheta_{j-k+1}\right)$ and $Z_{4 / 3}$ is defined in the original paper. The test statistic is more powerful than those based on multipower variation, but it is also affected by zero returns.

\section{Tests based on jump-time identification:}

The ABD-LM (The test compares standardized returns to a threshold. The null hypothesis is that there are no jumps at each time. The test is able to identify the exact time $i$ and the number of jumps within an intraday trading day $t$. The local volatility is estimated computing the bipower variation at each time interval, $B V_{t, i}$, choosing a window size $K$.

$$
\widehat{\sigma}_{t, i}^{2}=\frac{B V_{t, i}}{K-2}=\frac{\pi}{2} \frac{1}{K-2} \sum_{j=i-K+2}^{i-1}\left|r_{t, j}\right|\left|r_{t, j-1}\right|
$$

Thus, the standardized return is $\varsigma_{t, i}=r_{t, j} / \widehat{\sigma}_{t, i}$.

Since it is constructed using the bipower variation of BNS (2006), the distribution of $\varsigma_{t, i}$ is asymptotically standard normal when there are no jumps. The jump test is constructed using a threshold based on Gaussian extreme value theory $\max \left(\varsigma_{t, i}\right)$

$$
\frac{\max \left(z_{j}\right)-C_{n}}{S_{n}} \underline{L} \xi
$$

and $P(\xi)=\exp (-\exp (-x))$, where

$$
\begin{aligned}
C_{n} & =\frac{(2 \log n)^{1 / 2}}{0.8}-\frac{\log \pi+\log (\log n)}{1.6(2 \log n)^{1 / 2}}, \\
S_{n} & =\frac{1}{0.8(2 \log n)^{1 / 2}} .
\end{aligned}
$$

The choice of $K$ usually considers the number of trading days and the number of intervals within the trading day. When a jump occurs the size is $r_{t, j}$ and the jump component is defined as the sum of intraday squared returns, $J L M_{t}=\sum_{j=1}^{M} r_{t, j}^{2} I_{\left\{\varsigma_{t, i}>\varsigma\right.}$. Furthermore, $J L M_{t}$ is decomposed into positive and negative jumps, $\left.J L M_{t}^{+}=\sum_{j=1}^{M} r_{t, j}^{2} I_{\left\{r_{t, j}>0 \wedge \varsigma_{t, i}>\varsigma\right.}\right\}$

\footnotetext{
${ }^{6}$ In the empirical application we use several kernel density functions: Normal, Parzen, cosine and Epannechnikov. The choice of $c_{\vartheta}$ determines the jump detection. Choosing larger values of $c_{\vartheta}$ reduces the percentage of detected jumps. We have also tried with $c_{\vartheta}=\{4,5,6\}$.
} 
and $J L M_{t}^{+}=\sum_{j=1}^{M} r_{t, j}^{2} I_{\left\{r_{t, j}<0 \wedge \varsigma_{t, i}>\varsigma\right\}}$ (Patton and Sheppard, 2015). This statistic is only used for jump detection and cannot be used to decompose $R V$ into $J V$ and $C V$.

JO (2008) test uses $S w V_{t}(M)$ which is a function of the difference between arithmetic, $R_{t, j}=\left(p_{t, j+1}-p_{t, j}\right) / p_{t, j}$, and logarithmic, $r_{t, j}=\log p_{t, j+1}-\log p_{t, j}$, intraday returns

$$
S w V_{t}(M)=2 \sum_{j=1}^{M}\left(R_{t, j}-r_{t, j}\right)
$$

In the absence of jumps the difference between $S w V_{t}(M)$ and $R V_{t}$ is zero,

$\lim _{M \rightarrow \infty}\left(S w V_{t}-R V_{t}\right)=\left\{\begin{array}{cl}0 & \text { if no jumps in }[0, t] \\ 2 \int_{0}^{t}\left(\exp \left(J_{u}\right)-J_{u}-1\right) d q_{u}-\int_{0}^{t} J_{u}^{2} d q_{u} & \text { if there are jumps in }[0, t]\end{array}\right.$

Thus, jumps are captured in a exponential way. The corresponding JO test statistic is

$$
\frac{n B V_{t}}{\sqrt{\widehat{\Omega}_{S w V_{t}}}}\left(1-\frac{R V_{t}}{S w V_{t}}\right) \stackrel{L}{\rightarrow} N(0,1) \text { where } \widehat{\Omega}_{S w V_{t}}=3.05 \frac{M^{3}}{M-3} \sum_{i=0}^{M-4} \prod_{k+1}^{4}\left|r_{t, i+k}\right|^{3 / 2}
$$

The statistic takes very large values in the presence of high returns. Therefore, the statistic tends to overeject the null hypothesis of absence of jumps, that is jumps are usually over-identified. This statistic is only used for jump detection and cannot be used to decompose $R V$ into $J V$ and $C V$.

\subsection{Realized volatility models}

We propose to estimate several heterogenoeus autoregressive (HAR) models based on the initial work by Corsi (2009) for the $R V_{t}$ (HAR-RV). The HAR-RV model is a parsimoneous model because it reduces the number of parameters to be estimated. In particular, we propose the linear regression equation

$$
R V_{t}=\beta_{0}+\sum_{i=1}^{3} \beta_{i} R V_{i, t-1}+\varepsilon_{t}
$$

where $R V_{1, t-1}=R V_{t-1}, R V_{2, t-1}=\frac{1}{7} \sum_{t=1}^{7} R V_{t-1}$ and $R V_{3, t-1}=\frac{1}{30} \sum_{t=1}^{30} R V_{t-1}$. Then, with the decomposition into continuous and jump components for the different tests, we build the corresponding HAR-CV-JV model,

$$
R V_{t}=\theta_{0}+\sum_{i=1}^{3} \theta_{i} C V_{i, t-1}+\sum_{i=1}^{3} \lambda_{i} J V_{i, t-1}+\varepsilon_{t}
$$

where $C V_{1, t-1}=C V_{t-1}, C V_{2, t-1}=\frac{1}{7} \sum_{t=1}^{7} C V_{t-1}, C V_{3, t-1}=\frac{1}{30} \sum_{t=1}^{30} C V_{t-1}, J V_{1, t-1}=$ $J V_{t-1}, J V_{w, t-1}=\frac{1}{7} \sum_{t=1}^{7} J V_{t-1}$ and $J V_{m, t-1}=\frac{1}{30} \sum_{t=1}^{30} J V_{t-1}$. These models are estimated by ordinary least squares with heteroscedasticity and autocorrelation-corrected 
consistent standard errors to control for volatility clustering usually observed in time series analysis.

The assumption of identically and independently distributed Gaussian errors is tested. As Corsi et al. (2008) and Ciarreta and Zarraga (2016) show residuals can often show volatility clustering and probability distributions with excess kurtosis and skewness. Thus, we test for the presence of GARCH structures using the Ljung-Box (1978) statistic. Out of the several GARCH models proposed in the literature, we propose the following: $\operatorname{GARCH}(1,1)$ and $\operatorname{EGARCH}(1,1)$. The error term $\varepsilon_{t}$ is written as $\varepsilon_{t}=\sigma_{t} u_{t}$ where $u_{t}$ are independet and identically random standard normal distributed variables. The $\operatorname{GARCH}(1,1)$ models volatility as

$$
\sigma_{t}^{2}=\alpha_{0}+\alpha_{1} \varepsilon_{t-1}^{2}+\alpha_{2} \sigma_{t-1}^{2}
$$

where $\alpha_{0}>0, \alpha_{1} \geq 0, \alpha_{2} \geq 0$ and $\alpha_{1}+\alpha_{2}<1$ to ensure that the variance is positive and the process is stationary. The asymmetric $\operatorname{EGARCH}(1,1)$ is given by

$$
\log \sigma_{t}^{2}=\delta_{0}+\delta_{1} \frac{\left|\varepsilon_{t-1}\right|}{\sigma_{t-1}}+\delta_{2} \log \sigma_{t-1}^{2}+\delta_{3} \frac{\varepsilon_{t-1}}{\sigma_{t-1}}
$$

where there are no restrictions on the sign of the coefficients. The coefficient $\delta_{3}$ captures the asymmetric response of the volatility due to shocks of different sign: $\delta_{3}>0$ indicates an inverse leverage effect; $\delta_{3}<0$ indicates a direct leverage effect.

\subsection{Bias in the volatility measures}

We investigate the unbiasedness of the volatility measures following Jin and Maheu (2010) and Fang et al. (2012). We use daily data and estimate the following equation

$$
\widetilde{r}_{d}=\mu+\beta \sigma_{d, V} \epsilon_{d}
$$

where $\widetilde{r}_{d}=r_{d}-\sum_{j=1}^{N_{d}} \chi_{d, j}$ are the de-jumped returns, $\widetilde{r}_{d}$, which are equal to the return on day $d, r_{d}$, and $\sum_{j=1}^{N_{d}} \chi_{d, j}$ is the jump component. The jump component is the sum of all jumps within day $d, N_{d}$, of magnitude $\chi_{d, j}$. Finally, $\mu$ is the drift, $\sigma_{d, V}$ is the volatility on day $d$ estimated using volatility measure $V$ (BNS, ABD, ALM and CPR), $\epsilon_{d}$ is a Gaussian perturbation. The estimated parameter $\beta$ is expected to be one in the case of no-bias, and greater (less) than one when $V$ incorporates a downward (upward) bias.

\section{Japanese electricity market structure and regulatory frame- work}

The Japanese electricity industry used to be organized around 10 local monopolies. These local monopolies are vertically integrated Electricity Power Companies (EPCOs) that comprise generation, transmission and distribution. In addition, Japan has two 
types of frequency zones, $50 \mathrm{~Hz}$ and $60 \mathrm{~Hz}$, that are linked. EPCOs controll the bulk of the generation capacity. The process of liberation from these formerly verticallyintegrated fully-regulated electricity firms started in Japan in 1995 when the Electricity Utility Industry Law (EUIL) was passed that promoted the entrance of Independent Power Producers (IPPs). The target of the EUIL was to promote competition. Besides, it opened the possiblity of competitive bidding system creating a wholesale market. At the same time, the electricity rate system was revised, with the introduction of the yardstick assessment method for rate revision approval ${ }^{7}$, optional contract provisions, fuel cost adjustment system, and management efficiency review.

Further liberalization steps were taken in 2005 when the Japanese Electric Power Exchange (JEPX) was launched in April. JEPX operates as a pool and calculates the system marginal price for each of the areas. ${ }^{8}$ In the absence of congestions in the transmission grid, the price is the same. This is quite often the case in the areas that operate with the same frequency. The bidding is done by a uniform price auction system. Under this system, a bid is made for the combination of price and quantity of each product. A point of intersection where the buying and selling conditions comply with each other is sought, and the price and contract quantity are decided at this point. The purpose of a power exchange is to increase liquidity and flexibility in electricity trading, allowing market participants to fit their positions closer to delivery. The amount of electricity traded through JEPX has significantly increased although it remains at low levels (below 2\%).

There exists still a prominent position of these incumbents in the market. Table 1 illustrates this fact by showing the capacity mix of the EPCOs in 2013.

\footnotetext{
${ }^{7}$ Under yardstick regulation the performance a regulated utility is compared against of a group of comparable utilities (Shleifer, 1985). Thus, the price cap of each utility is determined in a way that can endup in losses. This system is expected to promote efficiency through cost cutting.

${ }^{8}$ The exchange operator does not open a real price trading. It opens a system marginal price for the operating regions and a virtual one for the whole country (asumming no restrictions in the network and no differences in the frequencies).
} 


\begin{tabular}{lcccccc}
\hline \hline \multicolumn{7}{l}{ TABLE 1. } \\
\hline EPCO & Frq. & TH & NU & HY & RES & Total \\
\hline 1. Hokkaido & 50 & 4.21 & 2.07 & 1.65 & 0.03 & 7.96 \\
2. Tohoku & 50 & 12.03 & 3.27 & 2.43 & 0.23 & 17.96 \\
3. Tokyo & 50 & 44.28 & 1.26 & 9.86 & 0.05 & 55.45 \\
\hline 4. Chubu & 60 & 24.02 & 3.62 & 5.50 & 0.03 & 33.17 \\
5. Hokuriku & 60 & 4.40 & 1.75 & 1.92 & 0.01 & 8.08 \\
6. Kansai & 60 & 19.41 & 8.93 & 8.23 & 0.11 & 36.68 \\
7. Chugoku & 60 & 7.80 & 0.82 & 2.91 & 0.01 & 11.54 \\
8. Shikoku & 60 & 3.45 & 2.02 & 1.15 & 0.01 & 6.63 \\
9. Kyusyu & 60 & 10.21 & 4.70 & 3.58 & 0.21 & 18.70 \\
\hline Total & & 129.81 & 28.44 & 37.23 & 0.69 & 207.38 \\
\hline \hline
\end{tabular}

Frq stands for frequency (Hz). Source FEPC (2014). TH, Thermal

NU, Nuclear; HY, Hydroelectric; RES, Renewable.

The total installed capacity of EPCOs is $208 \mathrm{GW}$ which represents around two thirds of the total installed capacity in the country. Limited transmission capacity between frequency zones (1200 MW) prevents prices to be exactly the same between zones. Generation mix has been diversified with a twofold target: (1) Reduction of carbon emissions to comply with international agreements, and (2) reduce dependence from international imports and fuel sources. Table 2 reports generation from different energy sources to total generation mix.

\begin{tabular}{ccccccc}
\hline \hline \multicolumn{2}{c}{ TABLE 2. } & \multicolumn{6}{c}{ Generation mix in 2013 (\%) } \\
\hline & NU & CO & LNG & OIL & HY & RES \\
\hline 2009 & 27.0 & 28.0 & 27.0 & 9.0 & 8.0 & 1.0 \\
2010 & 28.6 & 25.0 & 29.3 & 7.5 & 8.5 & 1.1 \\
2011 & 10.7 & 25.0 & 39.5 & 14.4 & 9.0 & 1.4 \\
2012 & 1.7 & 27.6 & 42.5 & 18.3 & 8.4 & 1.6 \\
2013 & 1.0 & 30.3 & 43.2 & 14.9 & 8.5 & 2.2 \\
\hline \hline
\end{tabular}

Source FEPC (2014). NU, Nuclear; CO, Coal;

LNG, Liquified Natural Gas; OIL, Oil-fired,

HY, Hydroelectric; RES, Renewable.

Generation mix is dominated by thermal plants which makes the country highly dependent on imports of fossil fuels. Only around $10 \%$ of total generation is from renewable sources. The perspectives to increase the share from hydroelectric sources is quite limited and up to the limit the country can reach, so it is not expected to significantly increase in the future. Renewable generation has not received the support as in other OECD countries such as Spain and Germany. The Fukushima Daiichi nuclear power plant accident in March of 2011 evidenced many problems of the electricity supply 
system that had to be corrected urgently. There exists little competition and strong price control. Little flexibility in changing the existing energy mix; hard to increase the ratio of renewable energy. Therefore, a deep electricity reform started to be promotting in a short-term horizon. Three regulatory advances in this line should be remarked: (1) Legal unbundling of the network by 2020, (2) retail liberalization by April 1st, 2016, and (3) renewable generation and distributed power generation promotion. Thus, smart metering should be fully accomplished in order to be successful to achieve (1) and (2) goals.

\section{Data and descriptive statistics}

Our data consists of 30 minute day-ahead price intervals for each day (48 intra-day observations). Data covers the period from April 2, 2005 until December 31, 2015. Therefore, we have 188, 448 half-hourly observations. Table 3 reports basic descriptive statistics of the price as well as by season and day of the week. ${ }^{9}$

\begin{tabular}{lcccc|ccccccc}
\hline \hline \multicolumn{1}{c}{ TABLE 3: Descriptive statistics of prices per season and day of the week } \\
\hline & Wtr & Spr & Sum & Aut & Mo & Tu & Wd & Th & Fr & All \\
\hline Min. & 4 & 3.30 & 2.95 & 4.34 & 2.95 & 4.00 & 3.99 & 3.78 & 3.83 & 2.95 \\
Max. & 42 & 35 & 60 & 39.23 & 39.67 & 44.92 & 43.67 & 55.00 & 60.00 & 60.00 \\
Mean & 11.90 & 10.89 & 10.93 & 11.09 & 11.50 & 11.66 & 11.64 & 11.72 & 11.67 & 11.20 \\
Median & 11.50 & 10.22 & 10.10 & 10.24 & 10.91 & 11.00 & 11.00 & 11.00 & 11.00 & 10.52 \\
St. Dv. & 4.67 & 4.07 & 5.02 & 4.08 & 4.56 & 4.72 & 4.72 & 4.86 & 4.87 & 4.49 \\
Sk & 1.07 & 0.59 & 1.68 & 0.91 & 0.89 & 1.06 & 1.09 & 1.44 & 1.41 & 1.18 \\
Kt (Ex.) & 5.60 & 2.84 & 9.98 & 4.62 & 4.73 & 5.57 & 5.63 & 8.74 & 8.73 & 6.84 \\
J-B & $5 \cdot 10^{6}$ & $7 \cdot 10^{5}$ & $2 \cdot 10^{7}$ & $7 \cdot 10^{6}$ & $5 \cdot 10^{6}$ & $6 \cdot 10^{6}$ & $5 \cdot 10^{6}$ & $9 \cdot 10^{6}$ & $1 \cdot 10^{7}$ & $8 \cdot 10^{7}$ \\
\hline \hline
\end{tabular}

Min, minimum; Max., maximum; St. Dv., Standard deviation; Sk, Skewness; Kt (Ex), Excess kurtosis J-B, Jarque-Bera test of normality.

The mean price is $11.20 ¥ / \mathrm{kWh}$ with a standard deviation of $4.49 ¥ / \mathrm{kWh}$, the minimum price is $2.95 ¥ / \mathrm{kWh}$ and the maximum price is $60 ¥ / \mathrm{kWh}$. Thus, the range of the price is quite large since large prices can be up to five times the mean price. Several seasonal effects are observed. Mean and median prices are higher in winter than in the rest of the seasons, probably due to house heating. Although air-conditioning consumption is high in summer it is not reflected in significantly higher prices than in other seasons maybe due to lower economic activity that offsets consumption. Day of the week seasonality is observed with lower mean, median and volatility of prices during weekends and Monday. The coefficient of skewness is positive and in many cases greater

\footnotetext{
${ }^{9}$ Descriptive statistics are also available for each zone upon request. For example, in the island of Honshu, the average prices in the Tohoku and Tokyo zones are $11.49 ¥ / \mathrm{kWh}$ and $11.44 ¥ / \mathrm{kWh}$, respectively, whereas the average prices in Hokuriku, Chobu, Kansai and Chugoku zones are around $11.18 ¥ / \mathrm{kWh}$. The rest of descriptive statistics follow a similar pattern.
} 
than 1 , thus the distribution is highly right-skewed. In all cases there is significant excess of kurtosis which indicates that the distribution of prices is leptokurtic. Jarque Bera test confirms that the distribution of prices is not normal.

Figure 1(a) plots the estimated kernel density against the standard normal ditribution to confirm the tests. Figure 1(b) plots the time series of prices.

[Insert Figure 1(a) and 1(b)]

Figure 1(a) shows how the distribution of prices is skewed to the left and bimodal. Figure 1(b) shows important price jumps in 2007/09/20, 2007/09/21, 2013/08/22, 2014/02/14, $2014 / 07 / 25$ and $2014 / 08 / 04$ to 07 . These larger prices are observed mostly during peak demand hours.

There exists a positive correlation between larger prices and traded quantities as demand effect dominates. However, this is not necesarily the case for returns. We control for return seasonality using two different filtering approaches. The first approach used by Chan et al. (2008), Ullrich (2012) and Ciarreta and Zarraga (2016), where the medians of the returns are subtracted from the returns for each month of the year, $m$, day of the week, $d$, and half-hour, $h h$, of the day to take into account the seasonal pattern effect observed in the descriptive analysis of the prices, $r_{t,(m, d, h h)}$. Therefore, the de-median returns are defined as $r_{t, j}^{* 1}=r_{t, j}-r_{t,(m, d, h h)}$. The second approach (REFERENCES) is using a nonlinear median smooth of size $p$ and obtain $r_{t, j}^{* 2}=\operatorname{median}\left(r_{t, j-p}, \ldots, r_{t, j-1}, r_{t, j}, r_{t, j+1}, \ldots, y_{t, j+p}\right)$. For $p=1$ is the usual definition of median. Both approaches yield to similar results since both are based on the same underlying median smoothing theory. Alternatively we could have filtered the data to remove the trend, $\tau$, and cyclical components, $c, \widetilde{r}_{t, i}=\tau_{t, i}+c_{t, i}$. The trend may be nonstationary, contain a deterministic or stochstic trend. We do not obtain large differences for the different filtering methods this is why we stick to the first approach. ${ }^{10}$

Table 4 reports descriptive statistics of the de-median returns $r_{t, j}^{* 1}$ as a whole and also by season and day of the week.

\begin{tabular}{lllll|llllll|l}
\hline \hline \multicolumn{1}{c}{ TABLE 4: Descriptive statistics of returns per season and day of week } \\
\hline & Wtr & Spr & Sum & Aut & Mo & Tu & Wd & Th & Fr & All \\
\hline Min. & -0.713 & -0.551 & -0.768 & -0.719 & -0.749 & -0.719 & -0.768 & -0.653 & -0.715 & -0.768 \\
Max. & 0.613 & 0.488 & 0.889 & 0.608 & 0.696 & 0.755 & 0.680 & 0.856 & 0.889 & 0.889 \\
Mean & -0.000 & -0.000 & -0.002 & 0.000 & -0.000 & -0.001 & -0.000 & -0.000 & -0.000 & -0.000 \\
Median & 0.000 & 0.000 & 0.000 & 0.000 & 0.000 & 0.000 & 0.000 & 0.000 & 0.000 & 0.000 \\
St. Dv. & 0.053 & 0.056 & 0.073 & 0.053 & 0.061 & 0.063 & 0.064 & 0.063 & 0.063 & 0.060 \\
Sk & 0.272 & 0.114 & 0.046 & 0.278 & 0.246 & -0.003 & -0.031 & 0.344 & 0.274 & 0.128 \\
Kt (Ex.) & 18.95 & 14.92 & 21.84 & 18.76 & 19.65 & 20.34 & 21.24 & 20.14 & 20.90 & 21.43 \\
JB & $4 \cdot 10^{5}$ & $2 \cdot 10^{5}$ & $6 \cdot 10^{5}$ & $5 \cdot 10^{5}$ & $3 \cdot 10^{5}$ & $3 \cdot 10^{5}$ & $3 \cdot 10^{5}$ & $3 \cdot 10^{5}$ & $3 \cdot 10^{5}$ & $3 \cdot 10^{5}$ \\
\hline \hline
\end{tabular}

On average adjusted returns are slightly negative. The largest return is 0.889 observed in summer which corresponds to a ratio of 2.5 between two consecutive prices

\footnotetext{
${ }^{10}$ In Appendix 1 we report summary statistics of the filtered returns for different filtering methods.
} 
(Friday 21, September, 2007 at half-hour 27). Thus, spikes are not as severe as those observed in other electricity markets worldwide. ${ }^{11}$ The percentage of zero returns is larger during weekends when market transactions are lower. There is no significant correlation between returns and quantity traded. Figure 2(a) plots the estimated kernel density against a standard normal ditribution of the returns and Figure 2(b) the time series of the returns.

$$
\text { [Insert Figure 2(a) and 2(b)] }
$$

Figure 2(a) clearly shows the excess of kurtosis and flatter than the normal distribution whereas Figure 2(b) shows the presence of return spikes above and below zero. Extreme returns are also associated frequently with price spikes as it is the case for the days 2007/09/20,2007/09/21, and 2013/08/22, although not for the rest of the days.

Risk managers do not like volatility because it involves complex hedging strategies and increases the risk of failures. Indeed, this is shown by the negative correlation between $R V_{t}$, defined in terms of the adjusted returns is used as our estimator of $I V_{t}$, and total quantity sold $(-0.1462) .{ }^{12}$ Table 5 summarizes the descriptive statistics of $R V$.

\begin{tabular}{lc}
\hline \hline \multicolumn{2}{l}{ TABLE 5: Descriptive statistics of RV } \\
\hline Min. & 0.0031 \\
Max. & 2.4296 \\
Mean & 0.1728 \\
Median & 0.1189 \\
St. Dv. & 0.1840 \\
Sk & 3.6120 \\
Kt (Ex.) & 25.2898 \\
J-B & $2.6 \cdot 10^{6}$ \\
\hline \hline
\end{tabular}

There exists skewness and excess of kurtosis, besides de Jarque-Bera test confirms thar the distribution is not normal. Figure 3(a) plots the estimated kernel density and the standard normal ditribution and Figure 3(b) plots the time series of the $R V$.

$$
\text { [Insert Figure 3(a) and 3(b)] }
$$

Figure 3(a) shows how a visual inspection of the distribution of $R V$ is not normal. Figure $3(\mathrm{~b})$ shows that there are few days when $R V$ presents sharp spikes. In particular, larger values are computed for 2007/09/20 and 2007/09/21, and also for 2011/08/19, $2011 / 08 / 31,2013 / 08 / 21,2013 / 08 / 29,2015 / 08 / 04$, and 2015/08/05. Thus, larger $R V$ is found during summer time days. Thus, a visual inspection justifies to test the presence of jumps using the tests proposed in Section 2. In order to restrict jumps, we choose

\footnotetext{
${ }^{11}$ For instance, in the EPEX day-ahead the ratio can be 25 times larger when prices are positive or in the Iberian market can be up to 7 times larger.

${ }^{12}$ In empirical applications two transformations are often used. One is the square root of the realized variance called properly realized volatility (see BNS, 2004) $\sqrt{R V}$, and another is the logarithmic transformation, $\log R V$. These transformations are used to smooth the time series. However, in our application we restrict to $R V$ because some of the tests are not suitable for any of these transformations.
} 
$\alpha=0.01$ as the significance level to detect jumps. For the BNS, MIN and MED tests, we choose 1-order processes. For the CPR test, we report results for the Gaussian kernel and $L=100$. Finally, for the ABD-LM we have that $K=\sqrt{365 \cdot 48}=132$. Table 6 reports the percentage of days with jumps detected by the different tests for adjacent returns and filtered returns. ${ }^{13}$

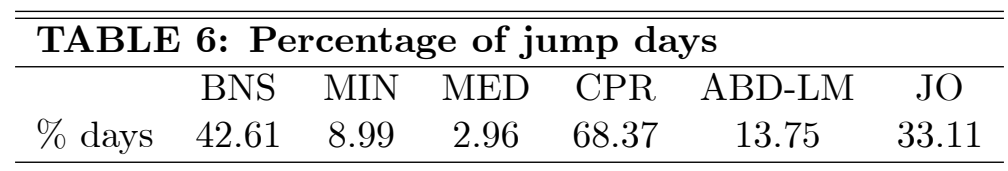

Note that the BNS test detects many jump days, $42.61 \%$ of the days are classified as jump days. By construction of the CPR test detects even more jump days. ${ }^{14}$ On the contrary, the MIN and MED tests are more conservative and detect around one fith and one tenth of jump days, respectively. ABD-LM and JO detect jump days inbetween the other tests. This is probably the result of having $25 \%$ of the observations with zero returns that tends to over-represent zero values in the calculations of $B V_{t}$ and $T Q_{t} .{ }^{15}$ Figure 4 plots JV for the different tests.

$$
\begin{array}{ll}
\text { [Insert Figure 4(a)] } & \text { [Insert Figure 4(b)] } \\
\text { [Insert Figure 4(c)] } & {[\text { Insert Figure 4(d)] }} \\
\text { [Insert Figure 4(e)] } & {[\text { Insert Figure 4(f)] }}
\end{array}
$$

Before we turn to the estimation of the models, we test for bias of the different intraday volatility measures used in this paper. The estimated equation of the returns against the different jump measures provides a test of the biasedness of the different approaches to the decomposition between continuous and jump components. Table 7 reports estimation results.

\begin{tabular}{lcccccc}
\hline \hline \multicolumn{7}{l}{ TABLE 7: Test for Bias } \\
\hline & BNS & MIN & MED & CPR & ABD-LM & RV \\
\cline { 2 - 7 }$\widehat{\mu}$ & 0.0012 & -0.0123 & $-0.0204^{* * *}$ & $0.0109^{* * *}$ & 0.0022 & $-0.0259^{* * *}$ \\
$\widehat{\beta}$ & $0.6074^{* * *}$ & $0.551^{* * *}$ & $0.566^{* * *}$ & $0.5812^{* * *}$ & $0.6016^{* * *}$ & $0.5756^{* * *}$ \\
\hline${ }^{* * *}$ & Significant at $1 \%$ level. & & & & \\
\hline
\end{tabular}

In all cases there is an upward bias in the volatility measures. The closest to no-boas $(\widehat{\beta}=1)$ is the BNS test followed by ABD-LM.

\footnotetext{
${ }^{13}$ Appendix 2 reports different jump detection results considering several specifications of the filtered returns and products of $k$-spaced adjacent returns. We have also analyzed other kernel functions for the CPR test but results do not change significantly.

${ }^{14}$ We have implemented the CPR test for values of $L=\{25,50,150\}$. For instance in the case of $L=150$, the percentage of jump days is 70.45. We have also considered $c_{\vartheta}=4$ and $c_{\vartheta}=5$ and the percentage of jump days reduces to $59.60 \%$ and $55.11 \%$, respectively.

${ }^{15}$ We have also considered the serial autocorrelation of the returns and using the staggered version of BNS the percentage of jump days decreases substantially. However, we continue considering this version in order to make results more comparable.
} 


\section{Estimation Results}

We estimate the different HAR models with and without the decompositions. Results are shown in Table 8 below.

\begin{tabular}{|c|c|c|c|c|c|c|c|}
\hline \multicolumn{8}{|c|}{ TABLE 8: HAR model estimation results } \\
\hline \multirow{9}{*}{$\begin{array}{l}\widehat{\beta}_{0} \\
\widehat{\beta}_{1} \\
\widehat{\beta}_{2} \\
\widehat{\beta}_{3}\end{array}$} & \multirow[t]{2}{*}{ RV } & \multicolumn{6}{|c|}{ CV-JV } \\
\hline & & & BNS & CPR & MIN & MED & ABD-LM \\
\hline & $0.0209^{* * *}$ & $\widehat{\theta}_{0}$ & $0.0186^{* * *}$ & $0.0185^{* * *}$ & $0.0210^{* * *}$ & $0.0212^{* * *}$ & $0.0144^{* * *}$ \\
\hline & $0.3417^{* * *}$ & $\widehat{\theta}_{1}$ & $0.3717^{* * *}$ & $0.3717^{* * *}$ & $0.3309^{* * *}$ & $0.3346^{* * *}$ & $0.3418^{* * *}$ \\
\hline & $0.3037^{* * *}$ & $\widehat{\theta}_{2}$ & $0.2919^{* * *}$ & $0.2913^{* * *}$ & $0.2969^{* * *}$ & $0.3040^{* * *}$ & $0.2612^{* * *}$ \\
\hline & $0.2334^{* * *}$ & $\widehat{\theta}_{3}$ & $0.2673^{* * *}$ & $0.2673^{* * *}$ & $0.2545^{* * *}$ & $0.2380^{* * *}$ & $0.2471^{* * *}$ \\
\hline & & $\widehat{\lambda}_{1}$ & $0.2936^{* * *}$ & $0.2936^{* * *}$ & $0.4122^{* * *}$ & $0.4921^{* * *}$ & $0.3011^{* * *}$ \\
\hline & & $\widehat{\lambda}_{2}$ & $0.3173^{* * *}$ & $0.3172^{* * *}$ & $0.3949^{* * *}$ & $0.3103^{* * *}$ & $0.3170^{* * *}$ \\
\hline & & $\widehat{\lambda}_{3}$ & $0.1971^{* *}$ & $0.1970^{* *}$ & 0.0372 & 0.0863 & $0.1812^{* * *}$ \\
\hline $\operatorname{adj-R^{2}}$ & 0.3786 & & 0.3796 & 0.3796 & 0.3798 & 0.3798 & 0.3805 \\
\hline AIC & -3977.8 & & -3981.0 & -3981.0 & -3982.6 & -3982.6 & -3981.1 \\
\hline
\end{tabular}

*** Significant at $1 \%$ level. ${ }^{* *}$ Significant at $5 \%$ level.

We only report the estimation results of the CPR test when the Gaussian-normal kernel is considered because results for the other kernel functions are very similar. Note that all the coefficients are positive and significant at the $1 \%$ level for the CV and showing strong volatility persistance. It is observed that the magnitude of the effect on $R V_{t}$ is larger from the previous day followed by the weekly and monthly average realized volatilities. This is due to the presence of volatility clusters decreasing over longer horizons. Considering the decomposition, it also shows high volatility persistance. In the case of the jump component, weekly effect is larger in magnitude than the previous day effect, and the monthly average effect is clearly smaller in accordance with the shortterm jump effect. Overall, based on adjusted $\mathrm{R}^{2}$ and AIC criteria the best model is the one that decomposes the realized volatility based on the approach by ABD (2010).

The error terms are analyzed in each of the estimated models in order to determine the presence of GARCH structures. In the case of RV, the Ljung-Box statistic on the correlations of the residuals and squares of residuals do not reject the hypothesis of the existence of GARCH effects in the residuals. Thus, we estimate the HAR-GARCH and HAR-EGARCH models for RV. 


\begin{tabular}{|c|c|c|c|c|c|c|c|}
\hline \multicolumn{8}{|c|}{ TABLE 9: HAR-GARCH model estimation results } \\
\hline \multirow{9}{*}{$\begin{array}{l}\widehat{\beta}_{0} \\
\widehat{\beta}_{1} \\
\widehat{\beta}_{2} \\
\widehat{\beta}_{3}\end{array}$} & \multirow[t]{2}{*}{ RV } & \multicolumn{6}{|c|}{ CV-JV } \\
\hline & & & BNS & CPR & MIN & MED & ABD-LM \\
\hline & $0.0105^{* * *}$ & $\widehat{\theta}_{0}$ & $0.0064^{* * *}$ & $0.0059^{* * *}$ & $0.0021^{* * *}$ & $0.0069^{* * *}$ & $0.0061^{* * *}$ \\
\hline & $0.3351^{* * *}$ & $\widehat{\theta}_{1}$ & $0.3668^{* * *}$ & $0.3676^{* * *}$ & $0.3475^{* * *}$ & $0.3413^{* * *}$ & $0.3268^{* * *}$ \\
\hline & $0.3876^{* * *}$ & $\widehat{\theta}_{2}$ & $0.3881^{* * *}$ & $0.3886^{* * *}$ & $0.3938^{* * *}$ & $0.4106^{* * *}$ & $0.3901^{* * *}$ \\
\hline & $0.1416^{* * *}$ & $\widehat{\theta}_{3}$ & $0.2472^{* * *}$ & $0.2565^{* * *}$ & $0.2244^{* * *}$ & $0.2098^{* * *}$ & $0.2341^{* * *}$ \\
\hline & & $\widehat{\lambda}_{1}$ & $0.2995^{* * *}$ & $0.3108^{* * *}$ & $0.3125^{* * *}$ & $0.3626^{* * *}$ & $0.3005^{* * *}$ \\
\hline & & $\widehat{\lambda}_{2}$ & $0.4340^{* * *}$ & $0.4273^{* * *}$ & $0.4956^{* * *}$ & $0.4045^{* * *}$ & $0.4245^{* * *}$ \\
\hline & & $\widehat{\lambda}_{3}$ & $0.1170^{* *}$ & $0.1316^{* * *}$ & -0.016 & -0.042 & $0.1133^{* *}$ \\
\hline$\widehat{\alpha}_{0}$ & $0.0004^{* * *}$ & $\widehat{\alpha}_{0}$ & $0.0004^{* * *}$ & $0.0004^{* * *}$ & $0.0004^{* * *}$ & $0.0004^{* * *}$ & $0.0004^{* * *}$ \\
\hline$\widehat{\alpha}_{1}$ & $0.2088^{* * *}$ & $\widehat{\alpha}_{1}$ & $0.2244^{* * *}$ & $0.0004^{* * *}$ & $0.2201^{* * *}$ & $0.2181^{* * *}$ & $0.2044^{* * *}$ \\
\hline$\widehat{\alpha}_{2}$ & $0.7904^{* * *}$ & $\widehat{\alpha}_{2}$ & $0.7789^{* * *}$ & $0.7982^{* * *}$ & $0.7820^{* * *}$ & $0.7837^{* * *}$ & $0.7689^{* * *}$ \\
\hline$A I C$ & -6613.262 & & -6618.556 & -6620.366 & -6612.484 & -6612.484 & -6611.511 \\
\hline
\end{tabular}

Note that $\widehat{\alpha}_{0}>0, \widehat{\alpha}_{1} \geq 0, \widehat{\alpha}_{2} \geq 0$ and $\widehat{\alpha}_{1}+\widehat{\alpha}_{2}<1$. According to the AIC the best model is the ABD-LM. Note that estimated coefficients do not much different in magnitude with respect to the simple HAR model. The significance of the GARCH parameters indicates that it is relevant to model volatility in the error term.

Finally, results from the HAR-EGARCH estimation are reported in Table 10.

\begin{tabular}{|c|c|c|c|c|c|c|c|}
\hline \multicolumn{8}{|c|}{ TABLE 10: HAR-EGARCH model estimation results } \\
\hline \multirow{8}{*}{$\begin{array}{l}\widehat{\beta}_{0} \\
\widehat{\beta}_{1} \\
\widehat{\beta}_{2} \\
\widehat{\beta}_{3}\end{array}$} & \multirow{2}{*}{\multicolumn{2}{|c|}{ RV }} & \multicolumn{5}{|c|}{ CV-JV } \\
\hline & & & BNS & $\mathrm{CPR}$ & Min & Med & ABD-LM \\
\hline & 0.0016 & $\widehat{\theta}_{0}$ & 0.0000 & 0.0000 & -0.0002 & -0.0005 & 0.0000 \\
\hline & $0.3517^{* * *}$ & $\widehat{\theta}_{1}$ & $0.3366^{* * *}$ & $0.3426^{* * *}$ & $0.3276^{* * *}$ & $0.3167^{* * *}$ & $0.3355^{* * *}$ \\
\hline & $0.5112^{* * *}$ & $\widehat{\theta}_{2}$ & $0.3627^{* * *}$ & $0.3558^{* * *}$ & $0.3734^{* * *}$ & $0.3836^{* * *}$ & $0.3427^{* * *}$ \\
\hline & 0.1267 & $\widehat{\theta}_{3}$ & $0.3398^{* * *}$ & $0.3433^{* * *}$ & $0.3247^{* * *}$ & $0.3103^{* * *}$ & $0.3298^{* * *}$ \\
\hline & & $\widehat{\lambda}_{1}$ & $0.2972^{* * *}$ & $0.3174^{* * *}$ & $0.2834^{* * *}$ & $0.3524^{* * *}$ & $0.2962^{* * *}$ \\
\hline & & $\hat{\lambda}_{2}$ & $0.4087^{* * *}$ & $0.4207^{* * *}$ & $0.4570^{* * *}$ & $0.3897^{* * *}$ & $0.4066^{* * *}$ \\
\hline & & $\widehat{\lambda}_{3}$ & $0.1848^{* * *}$ & $01711^{* * *}$ & 0.0005 & -0.0721 & $0.1748^{* * *}$ \\
\hline$\widehat{\delta}_{0}$ & $-0.1376^{* * *}$ & $\widehat{\delta}_{0}$ & $-0.1601^{* * *}$ & $-0.1548^{* * *}$ & $-0.1619^{* * *}$ & $-0.1625^{* * *}$ & $-0.1500^{* * *}$ \\
\hline$\widehat{\delta}_{1}$ & $0.0878^{* * *}$ & $\widehat{\delta}_{1}$ & $0.0585^{* * *}$ & $0.0614^{* * *}$ & $0.0633^{* * *}$ & $0.0521^{* * *}$ & $0.0577^{* * *}$ \\
\hline$\widehat{\delta}_{2}$ & $0.2777^{* * *}$ & $\widehat{\delta}_{2}$ & $0.3349^{* * *}$ & $0.3278^{* * *}$ & $0.3368^{* * *}$ & $0.3494^{* * *}$ & $0.3348^{* * *}$ \\
\hline$\widehat{\delta}_{3}$ & $0.9614^{* * *}$ & $\widehat{\delta}_{3}$ & $0.9545^{* * *}$ & $0.9559^{* * *}$ & $0.9542^{* * *}$ & $0.9536^{* * *}$ & $0.9544^{* * *}$ \\
\hline$A I C$ & -6604.21 & & -6608.55 & -6608.56 & -6615.468 & -6617.979 & -6608.58 \\
\hline
\end{tabular}

${ }^{* * *}$ Significant at $1 \%$ level. ${ }^{* *}$ Significant at $5 \%$ level.

We have estimated 15 non-nested models. To evaluate the in-sample performance of the models we select as preferred model the one with the minimum $A I C$ value. In this 
case, the best model is the HAR-GARCH-CV-JV with CPR Gaussian decomposition, followed by the HAR-GARCH-CV-JV with BNS decomposition. Thus, it is clearly relevant to decompose RV into continuous and jump component. We are interested in testing the robustness of our model. For that reason we also consider the Model Confidence Set approach by Hansen et al (2011).

\section{Conclusions, policy implications and further research}

The liberalization of the Japanese electricity market has implied the launching of a halfhourly Day-ahead market. Electricity subject to competitive market transactions is still marginal compare to the bulk of electricity consumed. However, the government following recommendations from regulatory agencies is trying to push further liberalization. As it happens in more developed electricity markets worldwide this trend tends to push prices down but volatility increases. As a response, agents implement hedging strategies following the experience of financial mrkets.

In this paper, we have shown how despite being marginal, there is volatility in the market that can be modelled using realized volatility. After implementing several test statistics, we show that there are jumps in volatility that can be best identified using the BNS approach. Using the HAR estimation, the nature of the price process is such that volatility depends on its own past. Including GARCH effects improves estimation results. Our results call for further research on the liberalization process of the Japanese electricity market. Even though we have identified the best model to explain volatility, differences in the jump detection call for further research on this initial part.

\section{References}

[1] Ait-Sahalia, Y., and Jacod, J., (2008), "Testing for jumps in a discretely observed process". The Annals of Statistics, 37, 184-222.

[2] Andersen, T. G. and T. Bollerslev (1998). "Answering the skeptics: yes, standard volatility models do provide accurate forecasts". International Economic Review 39, 885-905.

[3] Andersen, T. G., T. Bollerslev, and D. Dobrev (2007), "No-arbitrage semimartingale restrictions for continuous-time volatility models subject to leverage effects, jumps and i.i.d. noise: Theory and testable distributional implications". Journal of Econometrics 138, 125-180.

[4] Andersen, T. G., T. Bollerslev, and E. Schaumburg (2012), "Jump-robust volatility estimation using nearest neighbor truncation". Journal of Econometrics 169, 75-93.

[5] Asano, H., Okada, K, Yokohama, R., and Zhao, M., (2011), "Japanese approach to electricity deregulation policy - Modeling the spatial puchased power pricing". Discussion paper CRIEPI and TMU Japan. 
[6] Back, K. (1991). "Asset pricing for general processes". Journal of Mathematical Economics 20, 371-395.

[7] Bandorff-Nielsen, O., and Shephard, N., (2002), "Econometric analysis of realized volatility and its use in estimating stochastic volatility models". Journal of the Royal Statistical Society, Series B, 64, 253-280.

[8] Bandorff-Nielsen, O., and Shephard, N., (2004), "Power and Bipower Variation with Stochastic Volatility and Jumps". Journal of Financial Econometrics, Vol. 2, No. 1, $1-37$.

[9] Bandorff-Nielsen, O., and Shephard, N., (2006), "Econometrics of Testing for Jumps in Financial Economics Using Bipower Variation". Journal of Financial Econometrics, Vol. 4, No. 1, 1-30.

[10] Chan, K.F., Gray P., and van Campen, B., (2008), "A new approach to characterizing and forecasting electricity volatility". International Journal of Forecasting 24, 728-743.

[11] Ciarreta, A., and Zarraga, A., (2016), "Modeling realized volatility on the Spanish intra-day electricity market". Energy Economics, Volume 58, 152-163.

[12] Comte, F. and E. Renault (1998). "Long memory in continuous-time stochastic volatility models". Mathematical Finance 8, 291-323.

[13] Corsi, F., (2009), "A Simple Approximate Long-Memory Model of Realized Volatility". Journal of Financial Econometrics, 7, No. 2, 174-196.

[14] Corsi, F., Mittnik, S., Pigorsch, C., and Pigorsch, U., (2008), "The volatility of realized volatility". Econometric Reviews 27, 46-78.

[15] Corsi, F., Pirino, D., and Reno, R., (2010), "Threshold bipower variation and the impact of jumps on volatility forecasting". Journal of Econometrics 159, 276-288.

[16] Degiannakis, S., and Floros, C., (2016), "Intra-day realized volatility for European and USA stock indices". Working paper.

[17] Deng, S. (1999). "Stochastic models of energy commodity prices and their applications: Mean reversion with jumps and spikes". Unpublished manuscript, Georgia Institute of Technology.

[18] Dumitru, A-M., and Urga, G., (2012), "Identifying jumps in financial assets: A comparison between nonparametric jump tests". Journal of Business \& Economic Statistics, $30: 2$, 242-255.

[19] Escribano, A., Pena, J.I., and Villaplana, P. (2011). "Modeling electricity prices: International evidence". Oxford Bulletin of Economics and Statistics, 73, 5. 
[20] Fang, Y., Ielpo, F., and Sevi, B., (2012), "Empirical bias in intraday volatility measures". Finance Research Letters 9, 231-237.

[21] Federation of Electric Power Companies of Japan, (2015). Electricity Statistics Information.

[22] Geman, H., and Roncoroni, A., (2006), "Understanding the fine structure of electricity prices". The Journal of Business, 79, No 3, 1225-1261.

[23] Hosoe, Nobuhiro, (2006), "The deregulation of Japan's electricity industry". Japan and the World Economy 18, 230-246.

[24] Huang, X., and Tauchen, G. (2005), "The Relative Contribution of Jumps to Total Price Variance". Journal of Financial Econometrics, Vol. 3, No. 4, 456-499.

[25] Hull, J., and White, A., (1987), "The pricing of options on assets with stochastic volatilites." Journal of Finance, Vol. 42, 281-300.

[26] Hull, J., (2000), Options, Futures and other Derivatives, Prentice Hall.

[27] Ikeda, Shin, (2013), "An empirical microstructure analysis of the implied spread cost in the Japanese day-ahead electricity market". National Graduate Institute for Policy Studies, Tokyo, Japan.

[28] International Energy Agency, (2015), Statistics Energy Japan.

[29] Lee, S.S., and Mykland, P.A., (2008), "Jumps in financial markets: A new nonparametric test and jump dynamics". The Review of Financial Studies, 21 (6), $2535-2563$.

[30] Jiang, G.J., and Oomen, R.C.A., (2008), "Testing for jumps when asset prices are observed with noise - a "swap variance" approach". Journal of Econometrics 144, $352-370$.

[31] Liu, Z., Ma, F., Wang, X., and Xia, Z., (2016), "Forecasting the realized volatility: The role of junps". Applied Economics Letters, 23 : 10, 736-739.

[32] Ljung, G. M., and G. E. P. Box. (1978). On a measure of lack of fit in time series models. Biometrika 65, 297-303.

[33] Lucia and Schwartz

[34] G. M. Ljung; G. E. P. Box (1978). "On a Measure of a Lack of Fit in Time Series Models". Biometrika. 65 (2): 297-303.

[35] Makiko Nakanoa and Shunsuke Managi, (2008), "Regulatory reforms and productivity: An empirical analysis of the Japanese electricity industry". Energy Policy 36, 201-209. 
[36] Miyauchi, Hajime, and Misawa, Tetsuya, (2014), "Regression analysis of electric power market price of JEPX". Journal of Power and Energy Engineering, 2, 483488.

[37] Patton, A.J., and Sheppard, K., (2015), "Good volatility, bad volatility: Signed jumps and the persistence of volatility". The Review of Economics and Statistics, $97(3), 683-697$.

[38] Podolskij, M., and Ziggel, D. (2010), "New tests for jumps in semimartingale models". Statistical Inference for Stochastic Processes, 13, 15-41.

[39] Qu, H., Chen, W., Niua, M., and Li, X., (2016) "Forecasting realized volatility in electricitymarkets using logistic smooth transition heterogeneous autoregressive models". Energy Economics 54, 68-76.

[40] Skantze, P., Ilic, M., and Chapman, J., (2000), "Stochastic modelling of electric power prices in a multi-market environment", IEEE.

[41] Shleifer, A. (1985), "A Theory of Yardstick Competition," Rand Journal of Economics, Vol. 16, No. 3, 319-327.

[42] Ullrich, C.J., (2012), "Realized volatility and price spikes in electricity markets: The importance of observation frequency". Energy Economics 34, 1809-1818.

[43] Velleman, P. F. (1980), "Definition and comparison of robust nonlinear data smoothing algorithms". Journal of the American Statistical Association, 75, 609-615.

[44] Yamazaki, Takuya, (2015), "Japan's Electricity Market Reform and Beyond", Presentation of Agency for Natural Resources and Energy (ANRE), Ministry of Economy, Trade and Industry. 
Appendix: Filtering returns, realized volatility and jumps

\begin{tabular}{|c|c|c|c|c|}
\hline \multicolumn{5}{|c|}{ TABLE \#: Descriptive statistics of returns } \\
\hline & $r_{t, j}^{* 1}$ & $r_{t, j}^{* 2}(p=1)$ & $r_{t, j}^{* 2}(p=2)$ & $\widetilde{r}_{t, j}$ \\
\hline Min. & -0.768 & -0.9851 & -0.8659 & -0.7520 \\
\hline Max. & 0.889 & 0.9337 & 0.9729 & 0.7528 \\
\hline Mean & -0.0008 & 0.0003 & 0.0005 & 0.0000 \\
\hline Median & 0.0000 & 0.0000 & 0.0000 & 0.0000 \\
\hline St. Dv. & 0.060 & 0.0605 & 0.0635 & 0.0570 \\
\hline Sk & 0.128 & 0.4199 & 0.4910 & 0.4056 \\
\hline $\mathrm{Kt}(\mathrm{Ex})$. & 21.43 & 26.34 & 24.33 & 15.75 \\
\hline
\end{tabular}

\begin{tabular}{lcccc}
\hline \hline \multicolumn{5}{c}{ TABLE } \\
\hline & $r_{t, j}^{* 1}$ & $r_{t, j}^{* 2}(p=1)$ & $r_{t, j}^{* 2}(p=2)$ & $\widetilde{r}_{t, j}$ \\
Min. & 0.0031 & 0.0000 & 0.0012 & 0.0007 \\
Max. & 2.4296 & 2.5393 & 2.7758 & 2.5278 \\
Mean & 0.1728 & 0.1758 & 0.1937 & 0.1561 \\
Median & 0.1189 & 0.1172 & 0.1325 & 0.1068 \\
St. Dv. & 0.1840 & 0.1921 & 0.2082 & 0.1645 \\
Sk & 3.6120 & 3.3284 & 3.527 & 3.6179 \\
Kt (Ex.) & 25.2898 & 22.8928 & 24.7908 & 28.6389 \\
\hline \hline
\end{tabular}

\begin{tabular}{llllllll}
\hline \hline \multicolumn{7}{c}{ TABLE \#: } & \multicolumn{7}{c}{ Percentage of jump days } & & & \\
\hline \multirow{2}{*}{$r_{t, j}^{* 1}$} & $k=1$ & 42.61 & 8.99 & 2.96 & 68.37 & 13.75 & 33.11 \\
$r_{t, j}^{* 2}(p=1)$ & $k=2$ & & & & & & \\
$r_{t, j}^{* 2}(p=2)$ & $k=1$ & 49.41 & 3.59 & 2.54 & & & \\
& $k=2$ & & & & & & \\
$\widetilde{r}_{t, j}$ & $k=1$ & 61.11 & 7.54 & 2.17 & & & \\
& $k=2$ & & & & & & \\
& $k=1$ & 14.70 & 3.67 & 3.93 & & & \\
\hline \hline
\end{tabular}




\section{Technical appendix: Kernel functions}

A kernel density estimate is constucted using a weighted sum of values calculated using a kernel function $K(x)$ such that

$$
\widehat{f}_{K}=\frac{1}{q \cdot h} \sum_{i=1}^{n} w_{i} K\left(\frac{x-X_{i}}{h}\right)
$$

where $q=\sum_{i=1}^{n} w_{i}, h=\frac{0.9 m}{n^{1 / 5}}$ with $m=\min \left(\sqrt{\sigma_{x}^{2}}, \frac{i q r_{x}}{1.349}\right)$. Call $z=\frac{x-X_{i}}{h}$ then $K(z)$ can be one of these functions:

\begin{tabular}{ll}
\hline Cosine & $K(z)=\left\{\begin{array}{cc}1+\cos (2 \pi z) & \text { if }|z|<1 \\
0 & \text { otherwise }\end{array}\right.$ \\
\hline Epanechnikov & $K(z)=\left\{\begin{array}{cc}\frac{3}{4} \frac{\left(1-\frac{1}{5} z^{2}\right)}{\sqrt{5}} & \text { if }|z|<\sqrt{5} \\
0 & \text { otherwise }\end{array}\right.$ \\
\hline Gaussian & $K(z)=\frac{1}{\sqrt{2 \pi}} \exp \left(-\frac{z^{2}}{2}\right)$ \\
\hline Parzen & $K(z)=\left\{\begin{array}{cc}\frac{4}{3}-8 z^{2}+8|z|^{3} & \text { if }|z| \leq \frac{1}{2} \\
\frac{8(1-|z|)^{3}}{3} & \text { if } \frac{1}{2}<|z| \leq 1 \\
0 & \text { otherwise }\end{array}\right.$ \\
\hline Weibull & $K(z)=(1-p)^{z^{\beta}}-(1-p)^{(1+z)^{\beta}}$ \\
\hline
\end{tabular}


Technical appendix: Regression with Newey-West standard errors

The Newey-West standard errors for coefficients estimated by OLS regression. The error structure is assumed to be heteroskedastic and possibly autocorrelated up to some lag,

$$
\begin{aligned}
\widehat{\beta}_{O L S} & =\left(X^{\prime} X\right)^{-1} X^{\prime} y \\
\widehat{\operatorname{Var}}\left(\widehat{\beta}_{O L S}\right) & =\left(X^{\prime} X\right)^{-1} X^{\prime} \widehat{\Omega} X\left(X^{\prime} X\right)^{-1}
\end{aligned}
$$

The coefficients $\widehat{\beta}$ are those of OLS linear regression $\left(y=\beta^{\prime} X+e\right)$. The variance estimates of the estimated coefficients are calculated as

$$
X^{\prime} \widehat{\Omega} X=X^{\prime} \widehat{\Omega}_{0} X=\frac{n}{n-k} \sum_{i=1}^{n} \widehat{e}_{i}^{2} x_{i}^{\prime} x_{i}
$$

where $n$ is the number of observations, $k$ the number of regressors, and $\widehat{e}_{i}=y_{i}-x_{i} \widehat{\beta}_{O L S}$. If lags are included in the estimation then

$$
X^{\prime} \widehat{\Omega} X=X^{\prime} \widehat{\Omega}_{0} X+\frac{n}{n-k} \sum_{l=1}^{m}\left(1-\frac{l}{m+1}\right) \sum_{t=l+1}^{n} \widehat{e}_{t} \widehat{e}_{t-l}\left(x_{t}^{\prime} x_{t-l}+x_{t-l}^{\prime} x_{t}\right)
$$




\section{Technical appendix: Ljung-Box statistic}

The Box-Ljung test (1978) is a diagnostic tool used to test the lack of fit of a time series model. The test is applied to the residuals of a time series after fitting an $\operatorname{ARMA}(p, q)$ model to the data. The test examines $m$ autocorrelations of the residuals. If the autocorrelations are very small, we conclude that the model does not exhibit significant lack of fit. In general, the Box-Ljung test test the null hypothesis that the model does not exhibit lack of fit against the alternative hypothesis that the model exhibits lack of fit. Given a time series $Y$ of length $T$, the test statistic is defined as:

$$
Q=T(T+2) \sum_{k=1}^{m} \frac{\widehat{r}_{k}^{2}}{T-k}
$$

where $\widehat{r}_{k}$ is the estimated autocorrelation of the series at lag $k$ and $m$ the number of lags $q$. The null hypothesis is rejected if $Q>X_{1-\alpha, h}^{2}$, being $X_{1-\alpha, h}^{2}$ the chi-square distribution with $h=m-p-q$ degrees of freedom and significance level $\alpha$. The following table summarizes the Box-Ljung statistic for all the estimated models for $q=20$ :

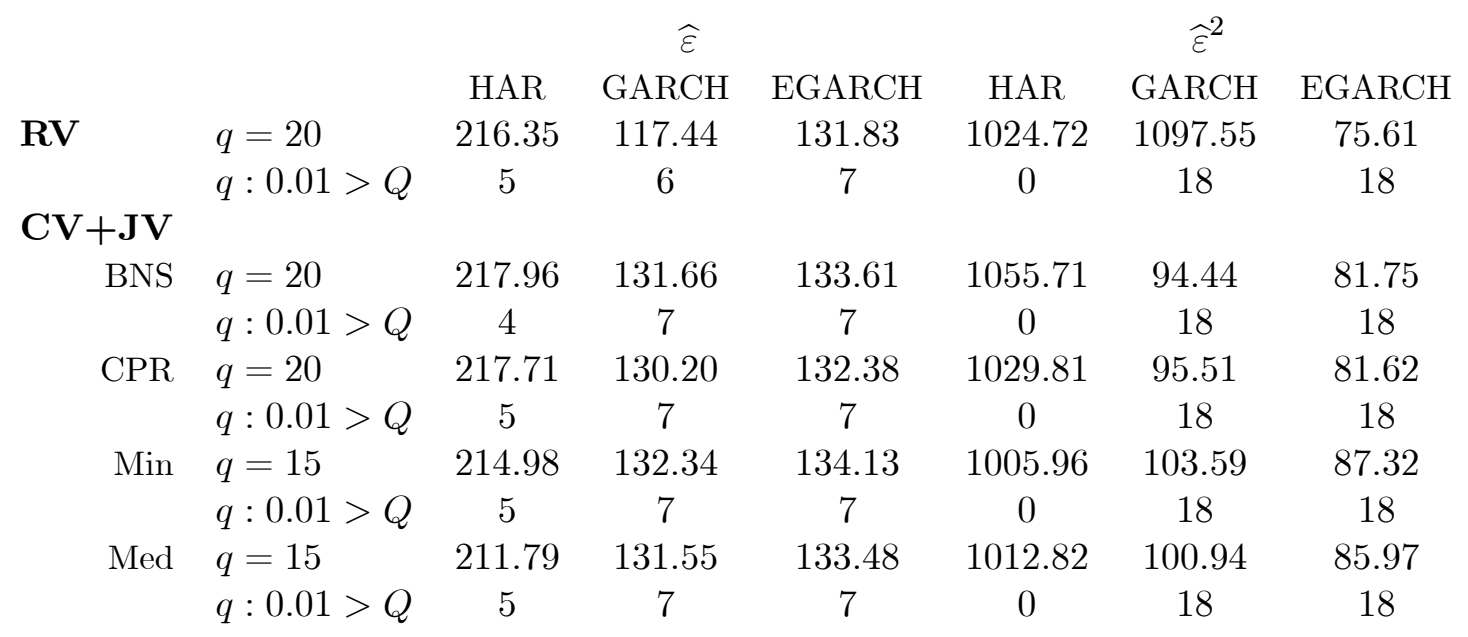




\section{Appendix: Quadratic Variation}

First, variation of a real-valued continuous function $f$ defined on an interval $[a, b] \subset$ $\mathbb{R}$ is a measure of the arclength of the curve with parametric equation $x \mapsto f(x)$ for $x \in[a, b]$. Thus, total variation of a real valued function $f$ defined on an interval $[a, b] \subset \mathbb{R}$ is defined as

$$
V(f)=\sup _{P} \sum_{i=1}^{n_{p}}\left|f\left(x_{i}\right)-f\left(x_{i-1}\right)\right|
$$

where the supremum runs over the set of all partitions of $[a, b], P=\left\{p=\left\{x_{0}, \ldots, x_{n_{p}}\right\} \backslash p\right.$ is a partition of $[a$ If $f$ is a differentiable function then $V(f)$ can be expressed as an integral,

$$
V(f)=\int_{a}^{b}|f(x)| d x
$$

Quadratic variation is a kind of variation process used to analyze stochastic processes such as martingales and semimartingales. Suppose now that $y_{t}$ is a real-valued continuous stochastic process defined on a probability space $(\Omega, F, P)$, the quadratic variation of the process, denoted as $[y]_{t}$, is defined as the sum of the squares of the differences of two consecutive numbers of the partition of $t$,

$$
[y]_{t}=\lim _{\|p\| \rightarrow 0} \sum_{i=1}^{n}\left(y_{t_{i}}-y_{t_{i-1}}\right)^{2}
$$

where $\|p\|$ is the norm or mesh of the partition. Recall that a partition of an interval $t$ on the real line is a finite sequence $x_{t}=\left(x_{t_{i}}\right)_{i=1}^{i=n}$ such that $x_{t_{0}}<x_{t_{1}}<\ldots<x_{t_{n}}$ where the norm is $\max \left\{\left|x_{t_{i}}-x_{t_{i-1}}\right|: i=1, \ldots, n\right\}$. A process $y_{t}$ has a finite varation if $\operatorname{prob}\left\{\left|x_{t_{i}}-x_{t_{i-1}}\right|<\infty\right\}=1$ for every $i=1, \ldots, n$. Note that by the definition it implies that the quadratic variation exists for all continuous finite variation processes and is zero. Instead suppose that $y_{t}$ is a real-valued non-continuous stochastic process defined on a probability space $(\Omega, F, P)$. If $y_{t}$ is a cadlag finite variation process then $y_{t}$ has left limit and it is right continuous, the quadratic variation can be expressed as

$$
[y]_{t}=\sum_{0<s \leq t}\left(\Delta y_{s}\right)^{2}
$$

It is important to relate quadratic variation to Ito processes. An Itô process is defined to be an adapted stochastic process that can be expressed as the sum of an integral with respect to Brownian motion, $W$, and an integral with respect to time of a predictable component, $\mu$,

$$
y_{t}=y_{0}+\int_{0}^{t} \mu_{s} d s++\int_{0}^{t} \sigma_{s} d W_{s}
$$

The quadratic variation of the process is

$$
[y]_{t}=\int_{0}^{t} \sigma_{s} d s
$$


Write the Ito process in the form of a differential equation:

$$
d y_{t}=\mu_{t} y_{t} d t+\sigma_{t} y_{t} d W_{t}
$$

then, by Ito's lemma, the stochastic process of any function $G(y, t)$ is

$$
d G(y, t)=\left(\mu y_{t} \frac{\partial G}{\partial y}+\frac{\partial G}{\partial t}+\frac{1}{2} \sigma^{2} y^{2} \frac{\partial^{2} G}{\partial y^{2}}\right) d t+\sigma y_{t} \frac{\partial G}{\partial y} d W_{t}
$$

where $\left(\mu y_{t} \frac{\partial G}{\partial y}+\frac{\partial G}{\partial t}+\frac{1}{2} \sigma^{2} y^{2} \frac{\partial^{2} G}{\partial y^{2}}\right) d t$ is the non-stochastic component and $\sigma y_{t} \frac{\partial G}{\partial y} d W_{t}$ is the stochastic component. Now let us consider the process with jumps,

$$
d y_{t}=\mu_{t} y_{t} d t+\sigma_{t} y_{t} d W_{t}+d J_{t}
$$




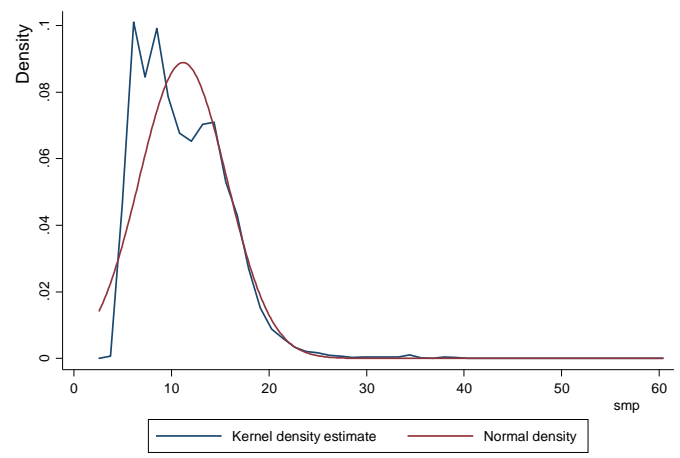

Figure 1(a).- Kernel density of smp and standard normal.
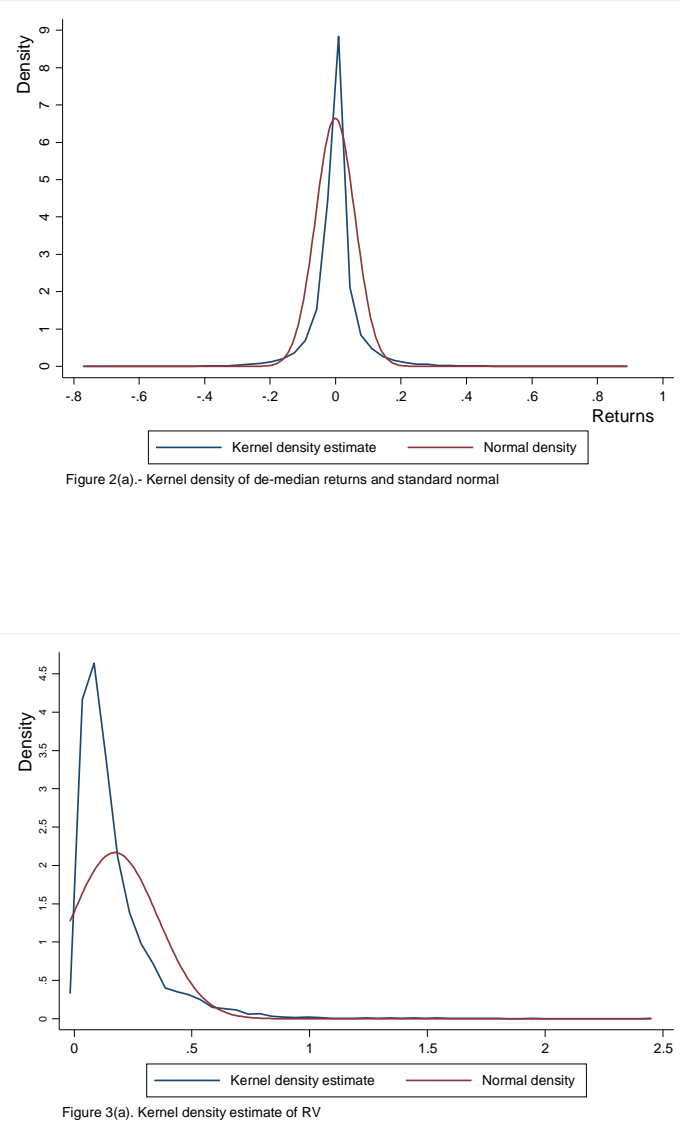
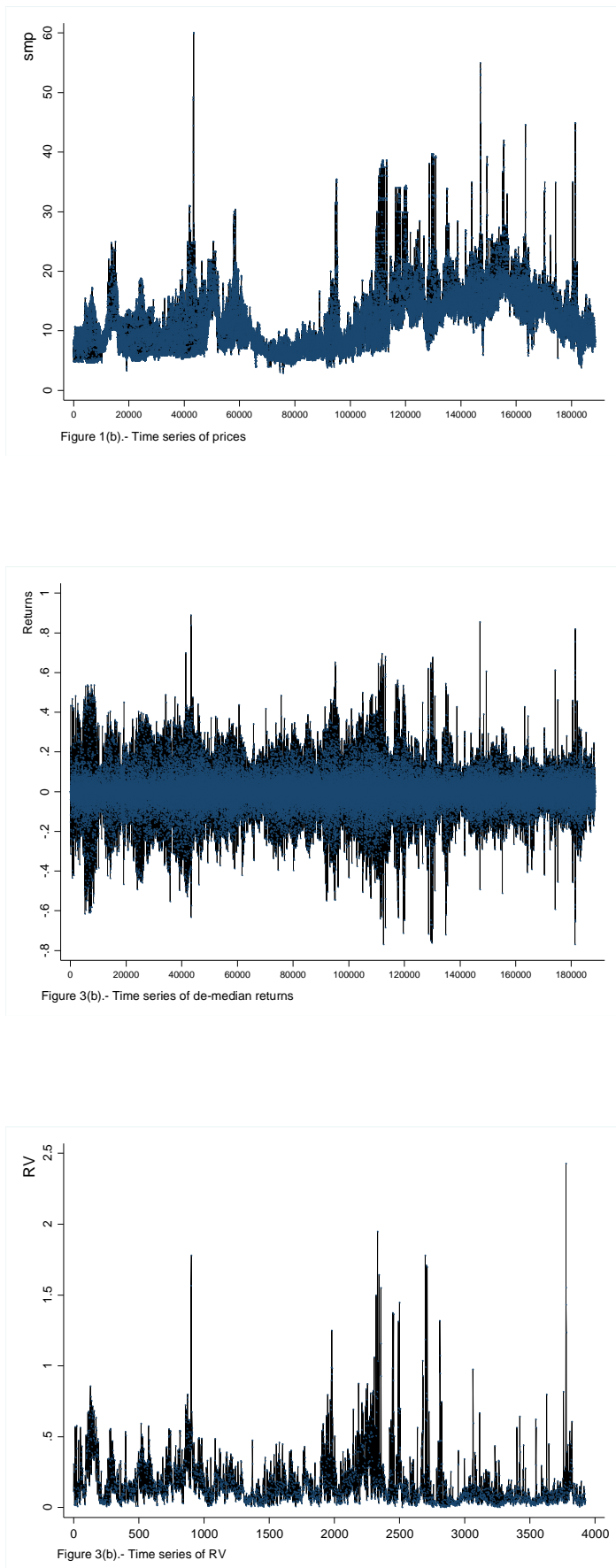

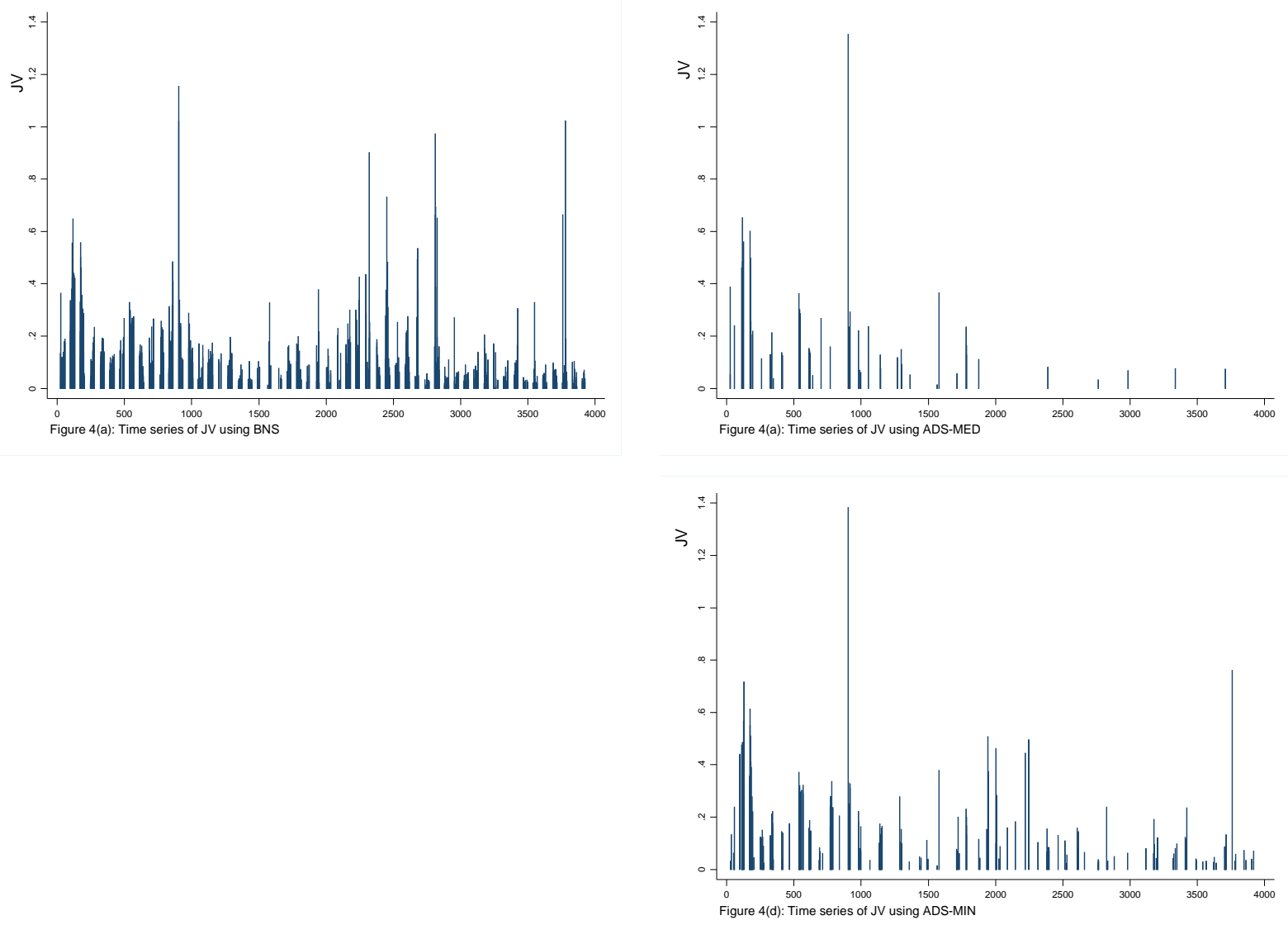